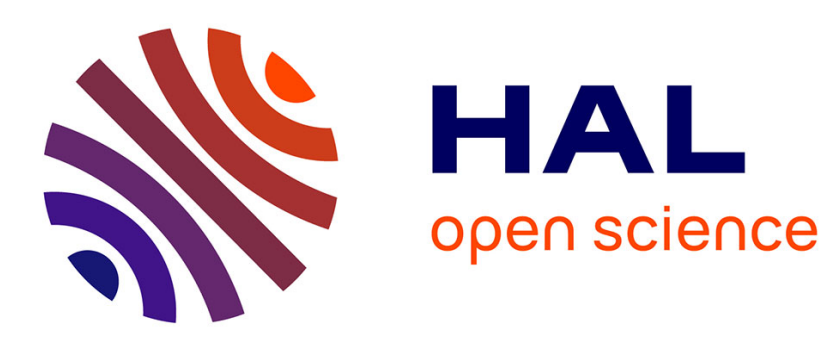

\title{
Simulation of the anisotropic behavior of titanium alloys during sheet metal forming
}

Benoît Revil-Baudard, Elisabeth Massoni

\section{To cite this version:}

Benoit Revil-Baudard, Elisabeth Massoni. Simulation of the anisotropic behavior of titanium alloys during sheet metal forming. 12th ESAFORM Conference on Material Forming, Apr 2009, Enschede, Netherlands. pp.Pages 73-76, 10.1007/s12289-009-0535-4 . hal-00509446

HAL Id: hal-00509446

https://hal-mines-paristech.archives-ouvertes.fr/hal-00509446

Submitted on 12 Aug 2010

HAL is a multi-disciplinary open access archive for the deposit and dissemination of scientific research documents, whether they are published or not. The documents may come from teaching and research institutions in France or abroad, or from public or private research centers.
L'archive ouverte pluridisciplinaire HAL, est destinée au dépôt et à la diffusion de documents scientifiques de niveau recherche, publiés ou non, émanant des établissements d'enseignement et de recherche français ou étrangers, des laboratoires publics ou privés. 


\title{
SIMULATION OF THE ANISOTROPIC BEHAVIOR OF TITANIUM ALLOYS DURING SHEET METAL FORMING
}

\author{
B. Revil-Baudard ${ }^{1 *}$ E. Massoni ${ }^{1}$ \\ ${ }^{1}$ Mines ParisTech, CEMEF - Centre de Mise en Forme des Matériaux, CNRS UMR 7635, BP 207, 1 \\ rue Claude Daunesse, 06904 Sophia Antipolis Cedex, France
}

\begin{abstract}
This paper introduces a constitutive elastoplastic model based on anisotropic yield criterion. An evolving anisotropy during simulation is considered. The anisotropy axes are updated with the deformation gradient. An anisotropic yield criterion, an isotropic hardening and a kinematic hardening model the plastic behavior of titanium alloys. Different plastic yield criteria are compared to show the accuracy of each plasticity model for the simulation of forming processes.
\end{abstract}

KEYWORDS: Anisotropy, Plasticity, Finite element method, Yield criteria, Hexagonal closed packed metals

\section{INTRODUCTION}

Understanding the anisotropic behavior is of primary importance for the sheet metal forming. Conditions and parameters of process depend on the direction of anisotropy. Modelling the anisotropic behavior of the titanium alloys is very important to make reliable simulations. The first yield criterion has been investigated by Hill [1]. This criterion is the simpliest one and it is implemented in most of the commercial softwares. Recently, new developments have been done and new non quadratic criteria have been developed [2]. Cazacu et al. [3] have extended these criteria to investigate the plastic behavior of closed packed materials. The aim of this paper is to develop an anisotropic elastoplastic model for titanium alloys.

To describe an evolving anisotropy, the rotation of the anisotropy axes must be updated at each increment. Working in an objective environment is crucial to simulate large deformations processes. Duchêne and al. [4] compared different axes rotations used in simulations. Based on their conclusions, a kinematic approach will be used in this paper. A physical approach at microlevel and based on the Mandel spin is in good agreement with experimental measurements. But the model presented in this paper describes plastic behavior at macroscopic level.

The implementation of a constitutive behavior in a FEM (Finite Element Method) software requires in our case the definition of an evolving anisotropic model coupled with different hardening laws. The stress tensor is updated at each integration point and a consistent tangent modulus is computed.

\section{EVOLVING ANISOTROPY}

Evolving anisotropy is a major issue to model properly the mechanical behavior of many materials. The following

\footnotetext{
${ }^{*}$ Corresponding author: 1 rue Claude Daunesse, 06904 Sophia Antipolis Cedex, France, benoit.revil@mines-paristech.fr
}

assumptions are made :

- the initial anisotropy is orthotropic, due to the rolling process. The initial orientation of the rolling direction is defined by $\gamma$.

- sheet metal processes only are considered in this study.

- the displacement during one increment is small and strains are considered as linear.

- all the behavior laws are expressed in the material basis, denoted by $\left\{a_{i}\right\}, \mathrm{i}=1 . .3$.

In this work, matrices are denoted by [.] and vectors by $\{$.$\} . The inverse and the transpose of a tensor are respec-$ tively given by $[.]^{-1}$ and $[.]^{T}$, the diadic product by $\otimes$. The implementation algorithm uses the Voigt notation to express a tensor with a vector.

Anisotropy axes have to be updated during the deformation. This is obtained, using either the deformation gradient $[\mathrm{F}]$ or the rigid rotation matrix $[\mathrm{R}]$, derived from the polar decomposition $[\mathrm{F}]=[\mathrm{R}][\mathrm{U}]$. The comparaison between $[F]$ and $[R]$ on the analytical evolution of axes during a simple shear test (fig.1) shows that the most accurate value is reached with the deformation gradient.

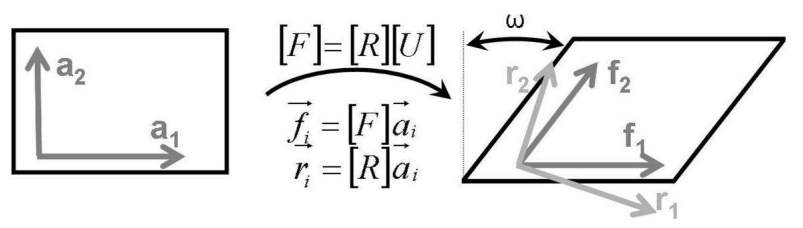

FIG. 1: Evolution of axis during simple shear test.

Anisotropy axes are updated during the deformation (fig.2) by the following relations :

$$
\begin{aligned}
& \left\{a_{i n+1}\right\}=\left\|[F]^{n}\left\{a_{i n}\right\}\right\| \quad i=1,2 \\
& \left\{a_{3 n+1}\right\}=\left\|\left\{a_{1 n+1}\right\} \wedge\left\{a_{2 n+1}\right\}\right\|
\end{aligned}
$$

Where $[F]^{n}$ is the deformation gradient for the $\mathrm{n}^{t h}$ in- 
crement. The in plane anisotropy axes $\left(\left\{a_{1}\right\},\left\{a_{2}\right\}\right)$ follow the sheet deformation and the third is normal to the metal sheet.

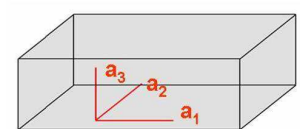

$\mathrm{n}^{\text {th }}$ increment

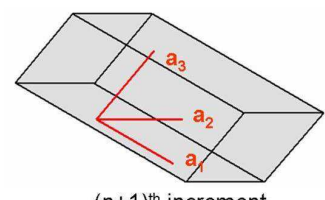

$(\mathrm{n}+1)^{\text {th }}$ increment
FIG. 2: Evolution of anisotropy axes during simulation.

\section{KINEMATIC CONSIDERATIONS}

The equilibrium equation is solved using finite elements in a reference basis $\left(0, e_{i}, \mathrm{i}=\mathrm{x}, \mathrm{y}, \mathrm{z}\right)$. To preserve objectivity, the stress increment $\Delta \sigma$ must be computed into the material basis $\left(0, a_{i}, \mathrm{i}=1 . .3\right)$. The transport between the material basis and the reference basis must be explicited to obtain the convergency. First, the bases and tensors are defined.

We first introduces a stationary cartesian coordinate system with an orthonormal basis $\left\{e_{i}\right\}$ for an Euclidean vector space, to define the stress and strain tensors in the body. Another vector basis $\left\{a_{i}\right\}$, as shown in fig. 3, describing the evolution of the anisotropy axes can be choose. The vector basis $\left\{a_{i}\right\}$ can be neither orthogonal nor normal and can be considered as a covariant basis for the Euclidean vector space. The stress tensor $[\sigma]$ is a contravariant tensor and the strain tensor $[\epsilon]$ is a covariant tensor.

In this work, we chose to express the stress tensor in local basis regarding recent work in composite structure [5] and older ones in thin sheet forming [6]. The tensor variance requires attention. To transport a tensor in an other basis, two operations are available, the push forward (from reference to current) and the pull back (from current to reference). Depending on the variance of the tensors, relations are not the same [7].

A transition matrix ([T].[M]) is computed to switch between the reference $\left\{\boldsymbol{e}_{i}\right\}$ and the material bases $\left\{\boldsymbol{a}_{i}\right\}$. Two changes of base are computed (fig.3), the first [M] between the global $\left(\left\{e_{i}\right\}\right)$ and one orthogonal basis $\left(0, \vec{a}_{1(i n t)}\right.$ , $\vec{a}_{3}$ ), and the second [T] to transport the tensors in the material basis $\left(0, \vec{a}_{i}\right)$. The first change of base transforms the axes $\left\{e_{z}\right\}$ into $\left\{a_{3}\right\}$. A first rotation of $\varphi$ around $\left\{e_{x}\right\}$ and a second of $\psi$ around $\left\{e_{y}\right\}$ are done. The first transition matrix $[\mathrm{M}]$ is :

$[M]_{\left(e_{x}, e_{y}, e_{z}\right) \rightarrow\left(a_{1 i n t}, a_{3}\right)}=\left[\begin{array}{ccc}\cos \psi & \sin \varphi \sin \psi & \cos \varphi \sin \psi \\ 0 & \cos \varphi & -\sin \varphi \\ -\sin \psi & \cos \psi \sin \varphi & \cos \varphi \cos \psi\end{array}\right]$

A First order development simplifies this matrix [M], which can be easily solved with the condition : $[M]\left\{a_{3}\right\}^{n}=\left\{a_{3}\right\}^{n+1}$. The second transition matrix [T] is expressed with the angle $\alpha$ and $\theta$ (fig.3), which are com- puted using vectorial and scalar product :

$$
[T]_{\left(a_{1 \text { int }}, a_{3}\right) \rightarrow\left(a_{1}, a_{1}, a_{3}\right)}=\left[\begin{array}{ccc}
\cos \alpha & \cos (\alpha+\theta) & 0 \\
\sin \alpha & \sin (\alpha+\theta) & 0 \\
0 & 0 & 1
\end{array}\right]
$$

FIG. 3: Transport of tensor between the reference $\left(\left\{e_{i}\right\}\right.$, $i=x, y, z)$ and the material basis $\left(\left\{a_{i}\right\}, i=1 . .3\right)$.

\section{MECHANICAL CONSIDERATIONS}

An elastoplastic model can be defined by different laws which are :

- an isotropic elastic law $[\mathrm{C}]:\{\epsilon\}=[C]\{\sigma\}$.

- a kinematic hardening law to define the kinematic tensor $[\mathrm{X}]$. An Amstrong and Friedrick kinematics law is chosen [8]: $\left\{\dot{X}_{i}\right\}=D_{i}\left\{\dot{\epsilon}^{p}\right\}-\xi_{i}\left\{X_{i}\right\}\left|\dot{\bar{\epsilon}}^{p}\right|$, where $\left\{\dot{\epsilon}^{p}\right\}$ is the plastic strain rate tensor and $\dot{\bar{\epsilon}}^{p}$ the equivalent plastic strain rate. This law associates a linear kinematic hardening term, characterized by $D_{i}$, and a recall term controlled by $\xi_{i}$ to model the dynamic recovery. Combining linear and non linear hardening allows a good modelling for small and large strains.

- a convex yield criterion to define the initial yield surface : $f(\sigma-X)$.

- a differentiable isotropic hardening law to define the flow stress $\sigma_{0}$.

The mechanical behavior is defined by the yield criterion $F_{p}$ :

$$
F_{p}=f(\sigma-X)-\sigma_{0}, \quad F_{p} \leq 0
$$

According to the value of $F_{p}$, the behavior could be elastic $\left(F_{p}<0\right)$ or elastoplastic $\left(F_{p}=0\right)$. This paper will focus on the plastic behavior, especially on the yield criterion shape. Two different criteria are implemented : the Hill 1948 one [1] and the Cazacu 2006 one [3].

\subsection{HILL 1948 CRITERION}

Hill developed a criterion, that can be expressed by the following relation with the stress deviator $[\mathrm{S}]$ :

$$
\begin{array}{r}
f(\{S\}-\{X\})=\left[\{S-X\}^{T}[H]\{S-X\}\right]^{1 / 2} \\
{[H]=\left[\begin{array}{cccccc}
G+H & -H & -G & 0 & 0 & 0 \\
-H & F+H & -F & 0 & 0 & 0 \\
-G & -F & F+G & 0 & 0 & 0 \\
0 & 0 & 0 & 2 N & 0 & 0 \\
0 & 0 & 0 & 0 & 2 M & 0 \\
0 & 0 & 0 & 0 & 0 & 2 L
\end{array}\right]}
\end{array}
$$

Where F, G, H, L, M, N are coefficients, identified by means of 6 experimental tests (tensile and shear tests). 


\subsection{CAZACU CRITERION}

Cazacu et al. [3] developed a criterion for the hexagonal closed packed metals. The plastic behavior is no more symmetric in tension and compression. This criterion is expressed as :

$$
f(\tilde{S})=\frac{1}{Q}\left(\sum_{i=1}^{3}\left(\left|\tilde{S}_{i}\right|-k \tilde{S}_{i}\right)^{a}\right)^{1 / a}
$$

where $\tilde{S}_{i}$ are the principal values of the tensor $\tilde{S}$, which may not be deviatoric. The parameter $a$ is a positive integer and $k$ a material constant. The yield function is convex for $a \geq 1$ and $-1 \leq k \leq 1$. These both parameters express the ratio of tensile versus compressive uniaxial yield stress. The tensor $\tilde{S}$ is obtained by :

$$
\left[\begin{array}{l}
\tilde{S}_{11} \\
\tilde{S}_{22} \\
\tilde{S}_{33} \\
\tilde{S}_{12} \\
\tilde{S}_{23} \\
\tilde{S}_{13}
\end{array}\right]=\left[\begin{array}{llllll}
L_{11} & L_{12} & L_{13} & & & \\
L_{12} & L_{22} & L_{23} & & & \\
L_{13} & L_{23} & L_{33} & & & \\
& & & L_{44} & & \\
& & & & L_{55} & \\
& & & & & L_{66}
\end{array}\right]\left[\begin{array}{l}
S_{11}-X_{11} \\
S_{22}-X_{22} \\
S_{33}-X_{33} \\
S_{12}-X_{12} \\
S_{23}-X_{23} \\
S_{13}-X_{13}
\end{array}\right]
$$

Where $S_{i j}$ are the stress deviator components and $L_{i j}$ are 9 material parameters. A constant parameter Q must be defined to normalize the yield criterion regarding the rolling direction :

$$
Q^{a}=\sum_{i=1}^{3}\left(\left|W_{i}\right|-k W_{i}\right)^{a}, W_{i}=\frac{2}{3} L_{i 1}-\frac{1}{3} L_{i 2}-\frac{1}{3} L_{i 3}
$$

Using this criterion implies the identification of 10 parameters, if $a$ is set to 2 . Numerous experimental tests have to be realized : 2 compressive tests, 2 tensile tests, 1 equibiaxial tension, 1 equibiaxial compression and 3 pure shear tests. Cazacu et al. have identified parameters for a titanium alloy $\left(4 \mathrm{A1}-1 / 4 \mathrm{O}_{2} 1 \%\right)$ [3]. The initial yield surface for this criterion (fig.4) shows the convexity of the anisotropic criterion. These values for the Cazacu 2006 criterion are used in the following part for the simulation.

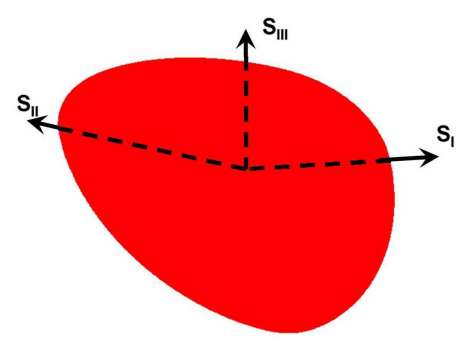

FIG. 4: Yield surface for the $4 A 1-1 / 4 O_{2}$ (1\%) titanium alloy plotted in the stress principal values basis.

\section{ANISOTROPIC ELASTOPLASTIC BE- HAVIOR IMPLEMENTATION}

This constitutive elastoplastic law is implemented into the FEM software Forge ${ }^{\circledR}$. The stress tensor and the tangent modulus are computed in the reference basis. In this part, all the values are expressed in the material basis. The transition matrices calculated in section 3 are used to switch between the global and the material bases. The subscripts ' $n$ ' and ' $n+1$ ' indicate the beginning and the end of one increment, and the subscript 'mat' means value in the material basis.

\subsection{STRESS INCREMENT COMPUTATION}

At the beginning of the increment, the strain rate tensor $\{\dot{\epsilon}\}^{n+1}$ and the previous stress tensor $\{\sigma\}^{n}$ are known. The anisotropy axes are updated with the deformation gradient. In a first trial the strains are considered as elastic and the stresses updated (elastic predictor) :

$$
\left\{\dot{\sigma}_{\text {elas }}^{n+1}\right\}=[C]^{-1}\left\{\dot{\epsilon}^{n+1}\right\}
$$

The yield criterion $F_{p}$ (equ.5) is updated. If $F_{p} \leq 0$, the behavior is elastic and the updated stress rate is the elastic one. Otherwise, an elastoplastic calculation must be done. The elastoplastic behavior is computed by the following relations :

$$
\left\{\begin{array}{l}
\left\{\dot{\epsilon}_{m a t}\right\}^{n+1}=[C]^{-1}\left\{\dot{\sigma}_{m a t}\right\}^{n+1}+\dot{\lambda}^{p} \frac{\partial f}{\partial\left\{\sigma_{m a t}\right\}^{n+1}} \\
F_{p}=f\left(\sigma_{m a t}-X_{m a t}\right)-\sigma_{0}=0
\end{array}\right.
$$

$\dot{\lambda}^{p}$ is the plastic multiplier. The stress increment evaluation depends on the chosen plasticity model : Hill 1948 or Cazacu 2006.

\subsubsection{Stress increment for Hill criterion}

Implementing the Hill criterion is direct. The increment stress provides :

$\left\{\sigma_{\text {mat }}^{n+1}\right\}=\left([I]+\Delta \lambda \frac{[C][H]}{\sigma_{0}}\right)^{-1}\left(\left\{\boldsymbol{\sigma}_{\text {mat }}^{n}\right\}+[\boldsymbol{C}]\left\{\Delta \epsilon_{\text {mat }}\right\}\right)$

where $[\mathrm{I}]$ is the unit matrix of size 6 . The yield criterion and the isotropic hardening can be easily re-written to have only one unknown $\Delta \lambda=\dot{\lambda}^{p} \Delta t . \Delta t$ is the time increment. The same formulation is used for strain increment $\left\{\Delta \epsilon_{\text {mat }}\right\}$. A Newton-Raphson scheme is sufficient to solve this equation.

\subsubsection{Stress increment for Cazacu criterion}

Solving the system (12) for the Cazacu 2006 criterion is more complex than the Hill criterion. First order development of equations of system (12) leads to correct the stress increment $\Delta \sigma$ and plastic multiplier $\Delta \lambda$.

$$
\begin{gathered}
d \Delta \lambda=\frac{F_{p}+\frac{\partial f}{\partial \sigma}[P]^{-1}\left(\{\Delta \epsilon\}-[C] \Delta \sigma-\Delta \lambda \frac{\partial f}{\partial \sigma}\right)}{\frac{\partial f}{\partial \sigma}[P]^{-1} \frac{\partial f}{\partial \sigma}+\frac{\partial \sigma_{0}}{\partial \Delta \lambda}} \\
d \Delta \sigma=[P]^{-1}\left(\{\Delta \epsilon\}-[C] \Delta \sigma-(\Delta \lambda+d \Delta \lambda) \frac{\partial f}{\partial \sigma}\right) \\
{[P]=\left([I]+\Delta \lambda[C] \frac{\partial^{2} f}{\partial \sigma^{2}}\right)^{-1}[C]}
\end{gathered}
$$


First and second derivatives of the yield criterion must be calculated at $\Delta \sigma^{n+1}$. Successive corrections on $\Delta \sigma$ and $\Delta \lambda$ are computed until convergency. To improve the algorithm, a line search procedure is adopted [9].

\subsection{ELASTOPLASTIC TANGENT MODULUS}

Forge ${ }^{\circledR}$ is based on an implicit solver. The tangent modulus $\frac{\partial \Delta \sigma}{\partial \Delta \epsilon}$ must be calculated. Differentiation of the yield criterion $F_{p}$ (equ.5) leads to a differential equation. Solving it gives an expression of the plastic multiplier $\Delta \lambda$. Finally combining this last equation and equation (1) of system (12) provides the linear tangent operator $\left[B_{\text {mat }}\right]$ :

$$
\left[B_{\text {mat }}\right]=[P]-\frac{[P] \frac{\partial f}{\partial \boldsymbol{\sigma}} \otimes \frac{\partial f}{\partial \boldsymbol{\sigma}}[P]}{\frac{\partial f}{\partial \boldsymbol{\sigma}}[P] \frac{\partial f}{\partial \boldsymbol{\sigma}}-\left(\frac{\partial \boldsymbol{\sigma}_{\mathbf{0}}}{\partial \bar{\epsilon}}+\frac{1}{\Delta t} \frac{\partial \boldsymbol{\sigma}_{\mathbf{0}}}{\partial \dot{\bar{\epsilon}}}\right)}
$$

To obtain the convergency of the general Newton Raphson scheme, the tangent modulus must be expressed in the global basis. The Relations explicited in section 3 are used.

\section{RESULTS}

The elastoplastic model presented in this paper has been validated with the elliptical bulging test [10]. Simulations with the Hill yield criterion have been performed for different orientations of the rolling direction. The initial anisotropic direction has a high importance in this test. According to this direction, the height of bulging and the equivalent plastic strains are not the same, but equivalent stresses are the same. Results for elliptical bulging test have been compared for the Cazacu 2006 criterion and the Hill 1948 criterion (fig.5). Use of a Cazacu 2006 criterion leads to the equivalent plastic strain localisation at the top of the ellipse.

\section{CONCLUSIONS}

A constitutive elastoplastic law has been implemented in Forge ${ }^{\circledR}$. This model is based on an evolving anisotropy, a kinematic hardening and an isotropic hardening. The anisotropy axes follow the deformation gradient. To remain objective, plastic strain tensor and stress tensor are computed in a material basis. The Hill criterion is simple to implement and the identification procedure for the material parameters could be done easily. The Cazacu 2006 criterion is more accurate, but its implementation is more difficult and 10 experimental tests are needed to identify all parameters. In the near future, our goal is to evaluate these two criteria on deep drawing simulations. Modelling the same material with these plasticity models would be done to compare the accuracy of numerical results with experiments.

\section{ACKNOWLEDGEMENT}

Financial support for this work from French fundation 'Fédération de Recherche pour l'Aéronautique et l'Espace" is gratefully acknowledged.

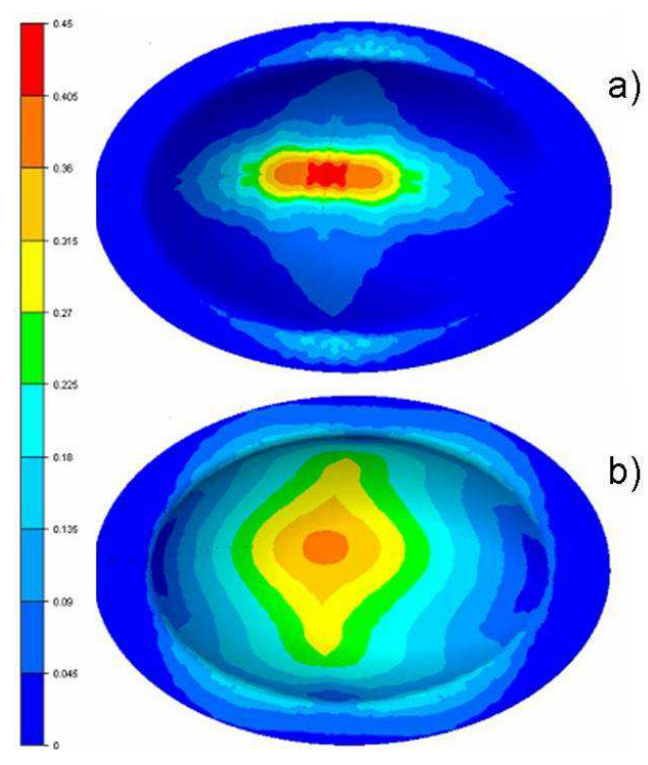

FIG. 5: Equivalent plastic strain for a bulging simulation :

a) Cazacu 2006 yield criterion ( $4 \mathrm{A1}-1 / 4 \mathrm{O}_{2}$ 1\%)

b) Hill 1948 yield criterion

\section{REFERENCES}

[1] R. Hill. Constitutive modelling of orthotropic plasticity in sheet metals. J. Mech. Phys. Solids, 38: 405-417, 1990.

[2] F. Barlat, J. W. Yoon, and O. Cazacu. On the linear transformations of stress tensors for the description of plastic anisotropy. Int. J. of Plasticity, 23: 876-896, 2007.

[3] O. Cazacu, B. Plunkett, and F. Barlat. Orthotropic yield criterion for hexagonal closed packed metals. Int. J. of Plasticity, 22:1171-1194, 2006.

[4] L. Duchêne, T. Lelotte, P. Flores, S. Bouvier, and A. M. Habraken. Rotation of axes for anisotropic metal in fem simulations. Int. J. of Plasticity, 24: 397-4427, 2008.

[5] X.Q. Peng and J. Cao. A continuum mechanics based non orthogonal constitutive model for woven composite fabrics. Composites Part A: Applied Science and Manufacturing, 36:859-874, 2005.

[6] K. Mattiasson. On the co-rotational finite element formulation for large deformation problems. $P H D$ Thesis Chalmers University of Technology, 1983.

[7] G. A. Holzapfel. Nonlinear Solid Mechanics : A continuum approach for engineering. Wiley, $1 \mathrm{st}$ edition, 2000.

[8] J. L. Chaboche. A review of some plasticity and viscoplasticity constitutive theories. Int. J. of Plasticity, 24:1642-1693, 2008.

[9] R. Knockaert. Numerical and experimental study of the strain localization during sheet forming operations. PHD Thesis ENSMP, 2001.

[10] M. Ben Tahar and E. Massoni. Use of the modified maximum force criterion for necking analysis during sheet bulging. 10th international Metal Forming Conference 2004, pages 229-234, 2004. 